African American/Black (28\%); Hispanic/Latino/x (25\%); Native Americans, First Nations, American Indian (0\%); Caucasian/ White (24\%); Multiracial (2\%); No Response (9\%). Feedback from research teams (aggregate of Strongly Agreed/Agreed responses) o ShARP panel was made up of relevant stakeholders (97\%) o ShARP session was worthwhile (100\%) o Stakeholder input will improve my research project (100\%) o I would engage stakeholders in future projects $(40 \%)$. Feedback from community stakeholders (aggregate of Strongly Agreed/Agreed responses) o ShARP session worthwhile (89\%) o I have an increased understanding of research after participating in this session (89\%) o Based on the experience, would consider providing input on a research study in the future (90\%) o This session was the first time I was asked to provide input on a research study (46\%) DISCUSSION/SIGNIFICANCE OF IMPACT: Preliminary results indicate engaging stakeholders in research can provide cultural and contextual adaptations that increase research relevance and feasibility in any phase of research. Conversely, stakeholders indicated an increased understanding of research. This poster will feature stakeholder and researcher perspectives. Increasing dialogues between research teams and community stakeholders can improve research design and relevance. The ShARPs programs aims to increase these types of dialogues which can be especially important for research teams who are unsure of who or how to begin engaging stakeholders in research. Gathering additional data via follow-up interviews will help us better understand the impact this program has on long term stakeholder engagement in research.

3578

Partnership Development: A learning community to advance institutional responsiveness to the opioid crisis in the city of Detroit and Wayne, County, Michigan

Karen D. Calhoun ${ }^{1}$, Laura Gultekin, PhD, FNP-BC ${ }^{1}$, Nikita Buckhoy, LMSW², Tinetra Burns, MS, RSST, CADC ${ }^{3}$, Zachary Rowe, $\mathrm{BA}^{4}$, Lisa Braddix, $\mathrm{MPH}^{5}$, Madiha Tariq, $\mathrm{MPH}^{6}$, Patricia Piechowski ${ }^{1}$, Donald R. Vereen Jr. ${ }^{1}$,

Dwight Vaughter, LMSW, ACSW, CAADC, CCS-M ${ }^{7}$ and

Kanzoni Asabigi, MD, $\mathrm{PhD}^{8}$

${ }^{1}$ University of Michigan; ${ }^{2}$ Connect Detroit; ${ }^{3}$ Detroit Wayne Mental Health Authority; ${ }^{4}$ Friends of Parkside; ${ }^{5}$ Greater Detroit Area Health Council; ${ }^{6}$ Arab Community Center for Economic and Social Services; ${ }^{7}$ Self-Help Addiction Rehabilitation (SHAR) and ${ }^{8}$ City of Detroit Health Department

OBJECTIVES/SPECIFIC AIMS: Facilitate relationships and partnership development to address the opioid crisis in Detroit and Wayne County Contribute to real-time conversations on opioid epidemic policy and practice to identify and build consensus on research questions Apply findings from each learning community session to policy briefs to better inform policymakers, providers and consumers; and advocate for institutional responsiveness METHODS/ STUDY POPULATION: The study population utilizes a purposive sampling approach to intentionally organize relationships and partnership development. For example, participants registered for the December 2018 session, "Detroit/Wayne County Opioid Crisis Learning Community Series: Data Session," include representation from school-based health clinics, community and faith-based organizations, health systems, city and county level public health, addiction/recovery organizations, law enforcement, academia and citizens. The team feels this approach ensures and builds diverse, team science perspectives and regional collaboration. The Detroit Area Mental Health Leadership Team formed in 2015 at a retreat held by the University of Michigan's Clinical and Translational Science Initiative attended by nearly 100 community-academic partners. Mental health, stigma and suicide were identified as community priorities by participants who attended the summit. A mental health workgroup formed and later expanded its membership to strengthen diverse perspectives. The team immediately designed and administered a survey amongst its partners creating the following priorities and focus: substance abuse interventions, healthcare access, and consumer awareness of mental health issues/available resources. Since data, policy and service are common threads to design interventions, the partnership decided to facilitate dialogue and discussion from the community on special topics related to the crisis, and share the community's recommendations on how to address them. The learning community series was designed as a bi-lingual format for sharing and expression. Deliberative democracy encourages inclusion of voices, interests and opinions often not heard or included in decisionmaking processes; driving the project's purposive sampling approach. Institutional responsiveness and advocacy for adoption of the community's recommendations will occur through strategic policy briefs summarizing each learning community session and the entire series. A dissemination plan will be utilized to encourage the policy briefs reach appropriate audiences for capacity building and institutional responsiveness. The learning community series will provide 5 sessions on data (impacting adolescents, emerging adults, and 20-mid 30 year-old adults), recovery/law enforcement, prescribing, and marijuana. The session topics arose from earlier assessment conducted by the Detroit Area Mental Health Leadership Team. RESULTS/ANTICIPATED RESULTS: A response to the opioid crisis should address community priorities identified through data, research and community input. Community providers should have access to real-time data and research to develop appropriate interventions and institutional responsiveness. Equally important is the need for legislators and others impacting resource allocation to hear from the community on priorities they feel should be addressed, and to better understand the need for new types of data and information to drive service delivery, policy and resources to address the crisis. The learning community series will focus on describing the epidemic and building infrastructure to collaborate, and share data and information to strengthen advocacy and responsiveness to address the crisis. We feel this will enable more efficient programming to strengthen service delivery that captures life experiences from those who directly interface with individuals impacted by the crisis. DISCUSSION/SIGNIFICANCE OF IMPACT: There is limited knowledge and consensus on types of data and information to effectively describe the opioid crisis. For example, data and information connecting gateway drugs such as marijuana with more hardcore drugs (i.e., opioids and heroin) is not available; community-based providers have limited access to what research says about the crisis; and local public and community providers are dependent upon the state for surveillance data. Individuals dealing with addiction and recovery often need immediate attention. A gap in access to services exists depending on types of insurance. For example, Medicaid and some HMOs require an assessment before clients can seek treatment, resulting in uncompensated care among providers to immediately address patients need. Access to healthcare is a longstanding issue in medically underserved communities. The impact of the crisis varies geographically in communities and regions due to cultural and ethnic differences, yet data and information on these differences is not readily available. Cultural competency and 
sensitivity is often an issue in medically underserved areas because stakeholders may feel professionals providing services do not relate to them effectively. Finally, the community does not understand the economic impact of the crisis. These issues make it difficult for community advocates and providers to work with elected officials, providers and others on the opioid crisis because they do not have the data and informed required to effectively flush out a hypothesis and form solutions. Information captured in the learning community series (i.e., presentations by experts, facilitated discussion and personal testimony) will be summarized in a policy brief after each session and the entire series. Recommendations and priorities from the community will be shared with providers, policymakers, the business community, consumers and others to provide community input on problem solving approaches, new interventions, types of data not currently available that should be captured, and other important strategies and information to address the crisis. This information will also encourage designing research questions to guide developing new community engaged and community based participatory research to address the crisis. Finally, utilizing a purposive approach in participant recruitment will encourage partnership development from a team science and capacity building perspective.

3430

\section{Promoting Stakeholder Research Competencies to Culturalize Health Science by the Miami CTSA: National Partnership for Training Community Health Workers in Patient-Centered Outcomes Research (PCOR) in 4 States Brendaly Rodriguez, MA, CPH \\ University of Miami Clinical and Translational Science Institute}

OBJECTIVES/SPECIFIC AIMS: To mobilize and engage CHWs/ promotores as stakeholders, we aim to promote the capacity for CHWs participate in patient centered research (PCOR) by locally implementing a structured research training curriculum for $\mathrm{CHWs}$. Main Questions: How the process of mobilization and engagement would be implemented at local/state level? What would be project challenges, risks and barriers at each and across sites? What modifications would be made to the initial PCOR for CHWs training curriculum and toolkit based on local feedback from collaborators, in both English and Spanish? What would be lessons learned on mobilization, engagement of, and sustainability for CHW training organizations as partners in PCOR? METHODS/STUDY POPULATION: Measures: Monthly calls and quarterly reports from local organizations on activities, deliverables status, modifications, project implementation challenges/barriers and solutions (experienced and potential) to achieve goals of training 10 PCOR CHW Champions and a total of $360 \mathrm{CHWs}$ /promotores trained in PCOR. Input from Note taker reports, Feedback and Evaluation Forms from training attendees at each session. Co-authorship of team members on dissemination activities (submissions for presentations, posters, blogs entries, webinars). Activities/Procedures: Via a highly participatory, consensus-driven decision-making approach, each of the organizations in the target states provide input into refining the toolkit for local use, select the training champions and develop $\mathrm{CHW} /$ promotor outreach and recruitment plans, and deliver the PCOR training to CHWs. In addition, the organizations will also set up a local stakeholder $\mathrm{CHW} /$ promotores advisory group. Environmental scan and literature review continues on training content areas to complement field experience in implementing the trainings at local sites. Project information tools developed (info sheet for local organizations, informational slide set to be used at calls and statewide seminars, a templates for flyer for training recruitment, agenda, certificates). FL and TN pilot of the translation of the curriculum, addressing problematic concepts and terms, collecting feedback forms eliciting input on terminology variations across Spanish speaking populations and literacy levels. Project Collaborators: Día de la Mujer Latina, a patient advocacy group in Texas with CHW trainings in several states and Puerto Rico Chula Vista Community Collaborative, a community health empowerment organization in Southern California Progresso Community Center, a Latino-focused health coalition based in Tennessee Florida Community Health Worker Coalition, a statewide partnership dedicated to the support and promotion of the CHW profession in Florida. RESULTS/ ANTICIPATED RESULTS: Results: Trainings per State - Y1 JanOctober 2018 Totals: 11 PCOR Champions and 252 PCOR-Trained CHWs in CA-TX-TN Y2 Plans (October 2018-September 2019): Will continue to collect $\mathrm{CHW}$ trainee demographic data, $\mathrm{CHW}$ certification and patient/patient advocate status. Will conduct qualitative and quantitative analysis of all quarterly reports, Note taker, and trainee Feedback and Evaluation forms. Will reach goal of 360 PCOR-trained CHWs in FL, CA, TX and TN. Will continue to submit abstracts on our story on building patient and stakeholder capacity to participate as partners in patient-centered outcomes research, engagement and mobilization, from topic generation through the dissemination of research results. From evaluation analysis of content and process measures, we will discuss sustainability strategies as shared learning collaborative. DISCUSSION/SIGNIFICANCE OF IMPACT: Challenges/Decision Points Y1: On Mobilization and Engagement: Across partners, different levels of readiness/ capacities/structures, and access to local resources Process application for request for content and guest instructor approvals to the Texas Department of State Health Services (DSHS) for CHW and $\mathrm{CHW}$ Instructor credit Co-branding of informational products (recruitment flyers, agendas, certificates), slides of Welcome section On Content and Curriculum Development: Address different levels of literacy Format (1-day vs spread, in person/online, prescriptive vs open) Clear distinction between service provision and research; paradigm shift of transfer of skills Inserting examples of unethical health research past activities with both African American and Hispanic/Latino populations Reinstating phases of clinical studies basic information Creating a study research design activity in Chapter $1 \mathrm{CHW}$ role as member of research team Adding PCORIfunded project summary fact sheet per state On translations: Diversity of nuances of meaning in vocabulary for concepts On sustainability: Different degrees of organizational and personal involvement Next for newly PCOR-trained CHWs Meaningfully engaging patients and other stakeholders is increasingly recognized as requisite to generate research findings that are trusted, meaningful, and useful to clinicians, patients, and their families. One key engagement strategy used for engaging specific patients/caregivers and patient advocates in underserved communities is leveraging our partnerships with Community Health Workers (CHWs). Moreover, there is a documented need for higher level of training skills to undertake activities such as community health assessments, program evaluations and clinical studies. University of Miami (UM) along FL partners has successfully developed a 7-hr specialized toolkit on patientcentered research for CHWs and trained $148 \mathrm{CHWs}$ /promotores (original plans were for 100) across the six major statewide regions. Evaluation data resulted in $100 \%$ of attendees responses' as excellent and $99 \%$ would recommend it to others. Qualitative comments included "even though the training was tailored for research, I feel 